\title{
Features of Electroplastic Effect in Alloys with Martensite Transformation
}

\author{
Vladimir Stolyarov ${ }^{1,2}$
}

Received: 4 January 2018/Revised: 23 February 2018/Published online: 25 May 2018

(C) The Chinese Society for Metals and Springer-Verlag GmbH Germany, part of Springer Nature 2018

\begin{abstract}
Influence of different electric current modes (pulse and direct) on occurrence of the electroplastic effect under uniaxial tension in the coarse-grained alloys with martensite transformations is investigated. The materials are shape memory $\mathrm{Ti}_{49.3} \mathrm{Ni}_{50.7}$ alloy and metastable austenite transformation-induced plasticity (TRIP) steel. The paper contains experimental results of current impact on the "stress-strain" curves of the material. It has been taken an experimental measurement of the sample temperature during the test. It is shown that the shape of a stress-strain curves and type of the serrate plastic flow, connected with the martensitic transformation and electroplastic effect, depend on the current modes. Impact of pulse current and direct current suppresses shape memory and TRIP effect.
\end{abstract}

Keywords Electroplastic effect · TRIP effect · Pulse current · Deformation · Martensite transformation · Tension

\section{Introduction}

Electroplastic effect (EPE) had been discovered about 50 years ago [1-3]. It has been investigated generally for pure mono- and polycrystals and single-phase alloys. EPE is displayed a considerable reduction in flow stresses and increase in deformability under the influence of electric current in such processes as tension, compression, metal forming. Researches have shown that the nature of EPE can be connected with such physical mechanisms as thermal effect, electronic wind, skin, pinch, magnetostrictive and others effects $[4,5]$. However, the relative contribution of these mechanisms in each case is unknown and can strongly differ and depend on microstructure, chemical and phase composition of alloys [6] and as well electroconductivity, thermal conductivity, etc. However, the role of electric pulses in the materials with different phase

Available online at http://link.springer.com/journal/40195.

Vladimir Stolyarov

vlstol@mail.ru

1 Mechanical Engineering Research Institute, Russian Academy of Sciences, M. Kharitonievsky, 4, Moscow, Russia 101990

2 National Research Nuclear University MEPhI, Moscow, Russia magnetic conductivity is not investigated. Any unavoidable heating generated by current causes many arguments as to prevailing effect: electroplastic or thermal [7]. Some papers $[8,9]$ have the calculation that in case of Grade 2 tension with pulse current a drop of stress associated with the thermal heating is $50-70 \%$ of the experimental one. It is concluded that the remaining part of the stress drop belongs to electron dislocation interaction. Paper [10] contains the calculation of an increase in temperature of the wire during its electric heating under states close to the experimental ones in [2]. It demonstrates that it may not be excluded any generation of temperature about several hundreds of grades with used in these experiments the duration pulse.

It is important to emphasize that over the past few decades it has been experimentally proved that the electrically assisted forming under deformation is able to increase the deformability of the majority of light alloys used in the automobile industry.

The influence of the martensite transformations induced by strain and the electric current modes on EPE manifestation is of special interest. It is well known that some of modern materials are capable to experience martensitic transformation induced by both temperature and plastic strain. Shape memory TiNi-based alloys and metastable austenite transformation-induced plasticity (TRIP) steels are one of them. But the type of martensitic 
transformation in these materials is not the same: reversible in TiNi but irreversible in TRIP steel.

$\mathrm{Ti}_{49.3} \mathrm{Ni}_{50.7}$ alloy is of explorer's interest of the explorers due to its unusual shape memory property which is caused by the reversible thermoelastic martensitic transformation. In the recent years, a great number of studies are dedicated to a various ways of impact on the structure of such materials with the aim to improve shape memory characteristics. One of such methods is the electrical current processing together with plastic deformation [11, 12]. However, the impact of the mechanism of electrical impulses on the material particularly with the martensite transformation is still unexplored till the bitter end, and a number of important technological characteristic is not investigated which restrains any industrial application of this method.

In metastable austenitic steels, plastic deformation causes the martensitic transformation $\gamma$ (fcc) $\rightarrow \alpha^{\prime}$ (hcp) known as TRIP effect. Many investigations have been devoted to the research of TRIP effect $[13,14]$. The most common presentation about development in metastable austenitic steels is in the review [13]. Except chemical composition, the grain size, strain degree and deformation temperature are the most important parameters controlling the phase transformation [14]. There is hardening due to transformation of austenite to martensite in the places of possible strain location. Completeness of the phase transformation depends on the position of deformation temperature in relation to critical points of $M_{\mathrm{s}}, M_{\mathrm{f}}, A_{\mathrm{s}}$ $A_{\mathrm{f}}$ and $M_{\mathrm{d}} .\left(M_{\mathrm{s}}, M_{\mathrm{f}}, A_{\mathrm{s}}, A_{\mathrm{f}}\right.$ are the starting and final transformation temperatures of martensite and austenite, correspondingly; $M_{\mathrm{d}}$ is deformation temperature above which no transformation happens.) In accordance with work [14], the majority of TRIP steels has the distinctive feature $M_{\mathrm{s}}$ $<273 \mathrm{~K}$, and $M_{\mathrm{d}}>\mathrm{RT}$. The increase in the strain degree or structure refinement up to nano- and ultrafine-grained state in metastable AISI 304L steel promotes an increase in martensite quantity and additional hardening; however, deformability decreases [14].

Applied importance of TRIP steels consists in substantial increase in not only strength but also plasticity due to lack of necking. In this connection, the choice of the methods and modes of heat treatment and deformation of TRIP steels become especially important. The known deformation way based on EPE was used a long time ago in order to decrease the deforming stresses into a number of hard-deformed and brittle alloys [3, 4]. At the same time, it is revealed that pulse current in the shape memory alloys with martensitic transformation can not only stimulate the shape memory effect, but also suppresses phase transformations [6]. The study of this phenomenon is very interesting for the metastable steels with TRIP effect. However, there is not a lot of papers about the influences of current on the deformation behavior of TRIP steel. The current introduction under deformation should decrease a contribution of microcracks to general elongation, increase the thermal activation and dislocation mobility and prevent the necking. A lack of improvement in the steel deformability may be explained with an availability of the EPE activation threshold of current density, for which iron is approximately $2 \times 10^{6} \mathrm{~A} / \mathrm{mm}^{2}$ [15]. It appears that the applied current densities are not enough, and they only engender a decline of the relative elongation to failure as a temperature under cold working. It is known that an increase in the test temperature, even by $100{ }^{\circ} \mathrm{C}$, reduces the strength of TRIP steel [15]. The objective of the paper [16] is to propose a combination of the gradient structure and TRIP effect to produce high strength and high ductility. It is described an experimental study of surface mechanical attrition on sheets of austenitic stainless steel AISI 316L, subject to uniaxial tension [17]. With a current density value of $5-20 \mathrm{~A} / \mathrm{mm}$ [2], the relative elongation to failure of steel is considerably reduced with an increase in density of the direct current. It has not been detected any changes in the properties at room temperature, as in the literature [18, 19], in case of supplemental cooling.

As for the current mode, the influence of the direct, alternating or pulse current capable to lead to different contributions of EPE, is also hardly studied [4].

This paper aims to study the impact of electrical current modes on the deformation behavior under tension of shape memory TiNi alloy and TRIP steel in coarse-grained state. It represents the results of the study of EPE and temperature of the sample in states of uniaxial tension affected by impulse and direct current in comparison with a currentless mode.

\section{Experimental}

The coarse-grained $\mathrm{Ti}_{49.3} \mathrm{Ni}_{50.7}$ alloy in strip form was used. The alloy was prepared by water quenching from $800{ }^{\circ} \mathrm{C}$. The characteristic temperatures were $M_{\mathrm{s}}=6{ }^{\circ} \mathrm{C}$, $M_{\mathrm{f}}=-31{ }^{\circ} \mathrm{C}, A_{\mathrm{s}}=18{ }^{\circ} \mathrm{C}, A_{\mathrm{f}}=67^{\circ} \mathrm{C}$ [12]. The uniaxial tension was carried out on samples with the length of $70 \mathrm{~mm}$ and the section area of $0.9 \mathrm{~mm}$ [2] under various current modes, providing for the minimum Joule heating: single pulses with the current density of $j=500 \mathrm{~A} / \mathrm{mm}^{2}$, pulse duration $\tau=100 \mu \mathrm{s}$; multipulse current $(j=20 \mathrm{~A} /$ $\mathrm{mm}^{2}, \tau=100 \mu \mathrm{s}$, pulse frequency $f=100 \mathrm{~Hz}$ ); direct current $\left(j=4 \mathrm{~A} / \mathrm{mm}^{2}\right)$. The current density value was selected in such a way that the sample heating was a minimum.

The TRIP steel with the chemical composition of (wt \%): $0.2 \mathrm{C}-15 \mathrm{Cr}-5 \mathrm{Ni}-3 \mathrm{Mo}-0.63 \mathrm{Si}-0.6 \mathrm{Mn}$ in strip form by $0.30-0.35 \mathrm{~mm}$ in thickness processed by cold rolling was 
used. The phase composition of the strip surface was: (1) austenite-martensite $(0.47 / 0.53)$; (2) only martensite. In the sample section, the volume fraction of martensite slightly decreases from the surface to the center. Tension tests were performed on the flat dog-bone-shaped samples at the rate of $1 \times 10^{-3} \mathrm{~s}^{-1}$ using electric current of density $(j)$ and pulse duration $(\tau)$ and are shown in Table 2 . The sample temperature was measured by a thermocouple with accuracy of $\pm 5{ }^{\circ} \mathrm{C}$ and by pyrometer CEM IR-99.

Single-current pulses were applied to the elastic and plastic deformation area corresponding to martensitic transformation. The multipulse current and direct current were applied from the beginning of load the material fracture. The phase composition of the sample surface was defined by X-ray diffraction (XRD) with accuracy of 5\% by means of a diffractometer Ultima IV, "Rigaku", on the deformed part of the sample far from the location of the failure.

\section{Results and Discussion}

\section{1 $\mathrm{Ti}_{49.3} \mathrm{Ni}_{50.7}$ Alloy}

Table 1 contains the sample temperature data under the introduction of different current modes. The temperature of the samples does not exceed 37 and $69{ }^{\circ} \mathrm{C}$ at the multipulse and direct current. Under the mode of single pulses, the temperature of the sample differs from the room temperature by $6{ }^{\circ} \mathrm{C}$ only.

Figure 1 contains diagrams of tension $\sigma-\varepsilon$ with current and without current of $\mathrm{Ti}_{49.3} \mathrm{Ni}_{50.7}$ alloy, respectively. In the without-current diagram (Fig. 1, curve 1) at the stress values of $165-210 \mathrm{MPa}$, it is visible horizontal area of phase transformation $A \rightarrow M$, with the length up to $\varepsilon=6 \%$. The appearance of this area is an evidence of the potential possibility of shape memory effect appearance. The slopes of the curve before and after the horizontal area are different and characterize the elastic modulus of the material in austenitic and martensite structural states.

The single pulses (Fig. 1, curve 2) lead to an appearance of the upward stress jumps, which amplitude $\Delta \sigma$ decreases from 150 to $12 \mathrm{MPa}$ with an increase in strain. The downward stress jumps, which are peculiar to EPE, are missed. The observed jumps are associated with reversible

Table 1 Sample temperature under current introduction

\begin{tabular}{ll}
\hline Current mode & $T\left({ }^{\circ} \mathrm{C}\right)$ \\
\hline Without current & 18 \\
Single pulses & 24 \\
Multipulse current & 37 \\
Direct current & 69 \\
\hline
\end{tabular}

martensitic transformation owing to local current heating. This conclusion is proven to be true that the measured temperature $T_{\text {def }}$ under tension with single pulses of current has been in the interval $A_{\mathrm{S}}-A_{\mathrm{F}}$ for the present alloy. In the area of plateau, relevant to martensitic transformation, all the stress jumps have almost the same amplitude. Stress at the end of each jumps per $40 \mathrm{MPa}$ higher and is entirely congruent with the stress value on the without-current curve, respectively, in the area of plateau and strain hardening. This fact proves out that the Joule heating at that current mode is minimal, and the average test temperature is only $6{ }^{\circ} \mathrm{C}$ higher than the room temperature.

Under tension with the multipulse current (curve 3), perhaps due to a great impulse frequency, the stress jumps are not observed any more. A small Joule heating increases the flow stress by $30 \mathrm{MPa}$ in the area of martensitic transformation and then leads to its decrease. Some stress jumps coincide with this stress from single-current impulses. It is interesting to note that the flow stress at the strain hardening stage of the formed martensite in case of tension with multipulse current is lower than that without current. This fact requires additional structural research, and perhaps it coincides with a different speed of strain hardening of martensite and residual austenite. Additionally, the use of multipulse current increases the elongation to failure by $5 \%$.

The comparison of the mechanical behavior under single- and multipulse current shows a decrease in the total stress (EPE is more than Joule heating) and stress jumps, in the case of a multipulse current EPE is absent, but the Joule heating is large. The direct current mode (curve 4) causes a double increase in transformation stress and a drastic reduction of the transformation area, apparently, associated with a higher sample temperature $T=69{ }^{\circ} \mathrm{C}$ (Table 1). After the short transformation area, it is observed some increased strain hardening typical for austenite. Note that the different current modes have no impact on the breaking stress (650-670 MPa), but lead to a decrease in the elongation that is particularly important for the direct current.

\subsection{TRIP Steel}

Tensile properties (Table 2) and stress-strain curves (Figs. 1, 2) with and without current application, are presented. We can observe that austenite-martensite steel under tension without current application presents high strength and ductility due to TRIP effect. The application of the single-current pulses, even of high density, does not have significant influence on strength characteristics. However, it promotes a decrease in the elongation directly related to the quantity of impulses.

The application of multipulse and direct current leads to a decrease in ultimate tensile strength (UTS) and yield 

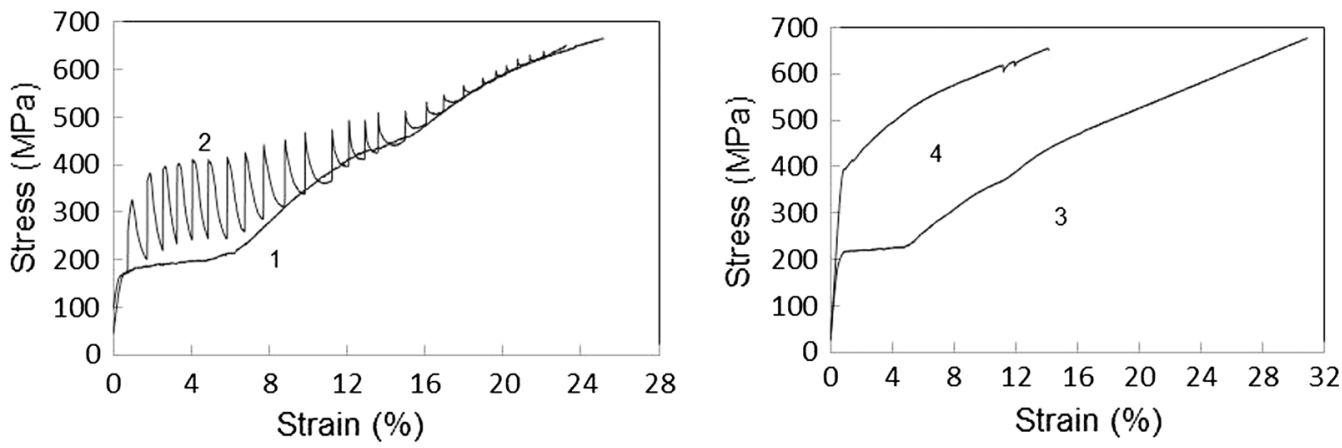

Fig. 1 Stress-strain curves for $\mathrm{Ti}_{49.3} \mathrm{Ni}_{50.7}$ alloy: 1 -without current; 2 - single pulses; 3 -multipulse current; 4 -direct current

Table 2 Mechanical properties of TRIP steel after tension with different current modes

\begin{tabular}{|c|c|c|c|c|c|c|}
\hline Phase state & Current regimes & Impact time $(s)$ & $T\left({ }^{\circ} \mathrm{C}\right)$ & UTS (MPa) & YS (MPa) & $\delta(\%)$ \\
\hline \multirow{4}{*}{$\mathrm{A}+\mathrm{M}$} & No current & - & RT & 1880 & 1830 & 27 \\
\hline & Single pulse, $j=460 \mathrm{~A} / \mathrm{mm}^{2}$ & $30 \times 10^{-3}$ & RT & 1780 & 1700 & 20 \\
\hline & Pulse current, $j=200 \mathrm{~A} / \mathrm{mm}^{2}, \tau=1 \times 10^{-3} \mathrm{~s}^{-1}$ & 80 & 150 & 1600 & 1400 & 2.3 \\
\hline & Direct current, $j=14 \mathrm{~A} / \mathrm{mm}^{2}$ & 80 & 200 & 1350 & 1000 & 2.4 \\
\hline \multirow[t]{4}{*}{ M } & No current & - & RT & 1200 & 1100 & 2.5 \\
\hline & Single pulse, $j=460 \mathrm{~A} / \mathrm{mm}^{2}$ & $6 \times 10^{-3}$ & RT & 1160 & 1100 & 1.7 \\
\hline & Pulse current, $j=200 \mathrm{~A} / \mathrm{mm}^{2}, \tau=1 \times 10^{-3} \mathrm{~s}^{-1}$ & 50 & 150 & 1160 & 1100 & 1.2 \\
\hline & Direct current, $j=14 \mathrm{~A} / \mathrm{mm}^{2}$ & 96 & 200 & 1170 & 550 & 3.0 \\
\hline
\end{tabular}

$R T$ room temperature
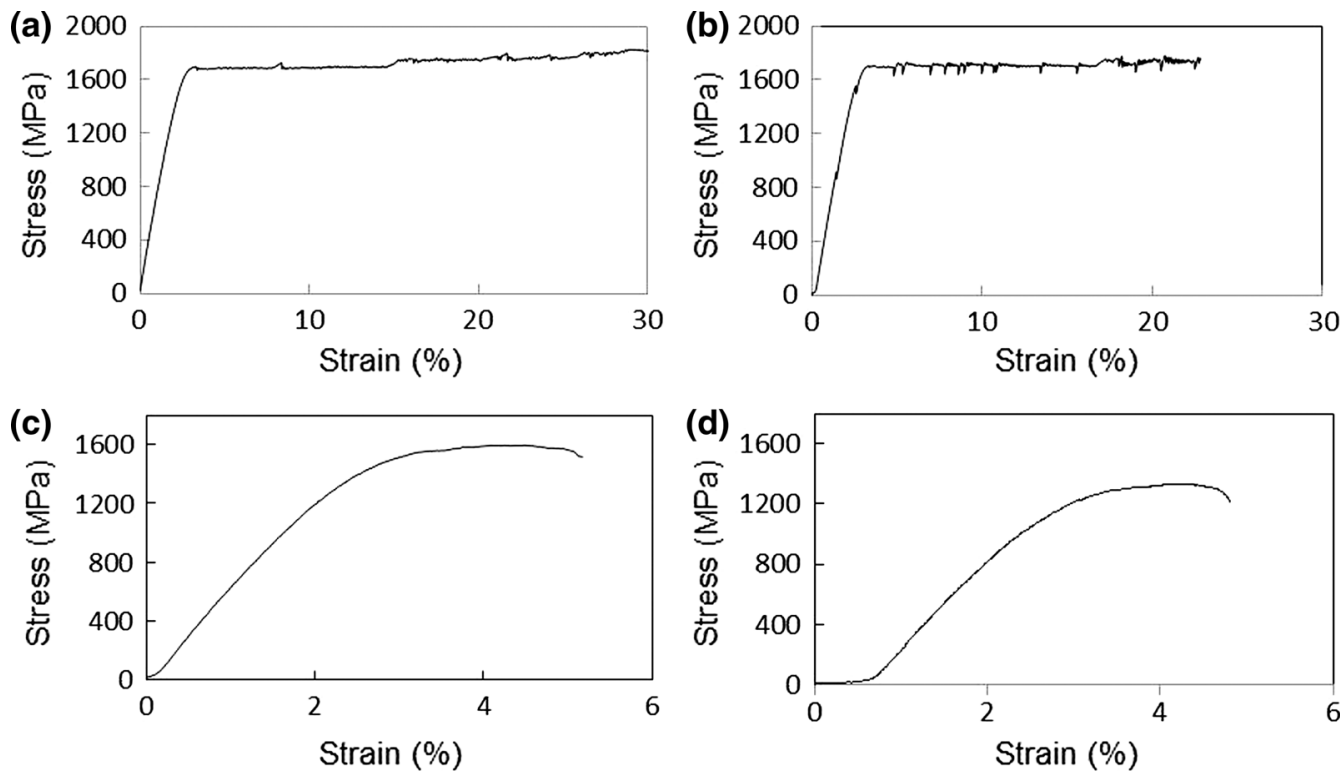

Fig. 2 Stress-strain curves of TRIP steel in austenite-martensite state: a without current; b single-pulse current; $\mathbf{c}$ multipulse current; $\mathbf{d}$ direct current

strength (YS) by $15-40 \%$ and, in particular, an abrupt decrease in the elongation. It has to be noted that the reduction in strength and plasticity presents a direct correlation with the increase in the current duration from microseconds up to several tenths of seconds and sample temperatures ranging from RT up to $150-200{ }^{\circ} \mathrm{C}$. This 
effect is probably related to the multipulse and direct current thermal impact, heavily amplified in the neck of the deformation. It is known that an increase in the test temperature, even by $100{ }^{\circ} \mathrm{C}$, reduces the strength of TRIP steel [16]. The lowest strength and elongation of steel correspond to the direct current mode.

TRIP steel in martensitic state under tension with and without current application has strength characteristics considerably below than in austenite-martensite state (Table 1), which indicates the absence of TRIP effect. Low elongation to failure for all current modes is connected with needle-shaped martensite grains and the limited number of sliding planes in the tetragonal lattice of martensite. It has been observed that, for both phases, the application of direct current, unlike pulse current, reduces drastically YS and prevents any plasticity reduction. Another reason for strength and plasticity decrease with the multipulse current application can be the cyclic softening/ hardening effect. As a result of the fatigue, provoked by the thermal and mechanical cycling, microcracks can be developed promoting the reduction in sample plasticity.

On the tensile curve without current, in austenitemartensite state, we can observe the serrated profile corresponding to plastic flow and low strain hardening (Fig. 2a). The presence of the serrated plastic flow (Luders bands) in TRIP steel is related to the deformation-induced $\alpha^{\prime}$-martensite making the neck creation more complex due to localized hardening.

Single-current pulses do not have a significant impact on the general shape of stress-strain curve but lead to occurrence of stress jumps downwards of amplitude ranging from 100 to $160 \mathrm{MPa}$ at the strain hardening stage (Fig. 2b). UTS of the samples tested without current application, and the one being the subject of single-current pulse application remains at the same level; however, single-current pulses produce a slight reduction in the elongation.

It would be possible to conclude that the presence of stress jumps downwards is connected with either local warming up or reverse transformation martensite to austenite.

However, as the flow stress at the strain hardening stage practically does not practically change, the phase composition should not significantly change as well. Available data for some materials indicate a weak thermal effect induced by the application of single-current pulses, in particular less than $20-40{ }^{\circ} \mathrm{C}$ [6]. Thus, stress jumps downwards on the tensile curves are the result of EPE. On the other hand, it is not clear to what phase EPE corresponds as the TRIP steel is in two-phase state. It is possible to assume that EPE appears in the martensite phase (stress jumps downwards), but TRIP effect appears in the austenite phase (stress jumps upwards). We can observe that the stress jumps also appear in the elastic area (Fig. 2b), the given fact is in contradiction to the expected dislocation mechanism of EPE [4].

The shape of stress-strain curve under the multipulse or direct current application changes sharply (Fig. 2c, d). The stress-strain curves have a typical shape for the stable austenite steel, with the developed stage of strain hardening and strain localization (necking). The stress jumps are not registered because of small pulse duration and large pulse frequency (Fig. 2c). Similar changes can be explained by significant thermal effect of current. The temperatures measured on the sample surface for the current regimes raise up to $150-200{ }^{\circ} \mathrm{C}$, and the local temperature inside of the samples can be still above and corresponds to ratio $T_{\mathrm{d}}>M_{\mathrm{d}}\left(T_{\mathrm{d}}\right.$-deformation temperature). It is known that in case of the deformation temperature rises more than $300{ }^{\circ} \mathrm{C}$, the strength and ductility of TRIP steel decrease sharply. It is connected with stabilization of the austenite to martensitic transformation as the transformation driving force decreases.

In martensite TRIP steel, the strain hardening is practically absent from the tensile curve produced without current; therefore, strength and ductility are noticeably lower in comparison with TRIP steel in austenite-martensite state (Fig. 3a).

The application of single-current pulses does not change the general shape of the tensile curve, but leads to occurrence of stress jumps downwards with the amplitude of $60 \mathrm{MPa}$ connected with EPE in martensite (Fig. 3b). For austenite-martensite steel, stress jumps appear not only in the flow stage but also in the elastic area that contradicts with the EPE mechanism based on the dislocation movement [4]. The general shape of the tensile curve and the softening stage (necking) at multipulse current introduction are kept (Fig. 3c) but stress jumps are not observed, as well as in austenite-martensite TRIP steel.

The X-ray investigations of austenite-martensitic TRIP steel have shown that tension without current increases the martensite volume fraction from 47 up to $82 \%$. After tension with single-current pulses and direct current, the martensite volume fraction decreases to 72 and $50 \%$, respectively, that corresponds to reduction in TRIP effect.

The above-presented results have shown that the deformation behavior at tension of both alloys shows strong sensitivity to the type and the modes of current. Really, under the influence of current there is an unusual hardening in the shape memory alloy or degradation of TRIP effect in the metastable steel. It is supposed that it is caused by the insignificant deformation temperature shift in relation to the upper range of a temperature interval of the reverse martensitic transformation. At the same time, the various cases depending on a martensitic transformation type and the current modes are possible. In alloy with 

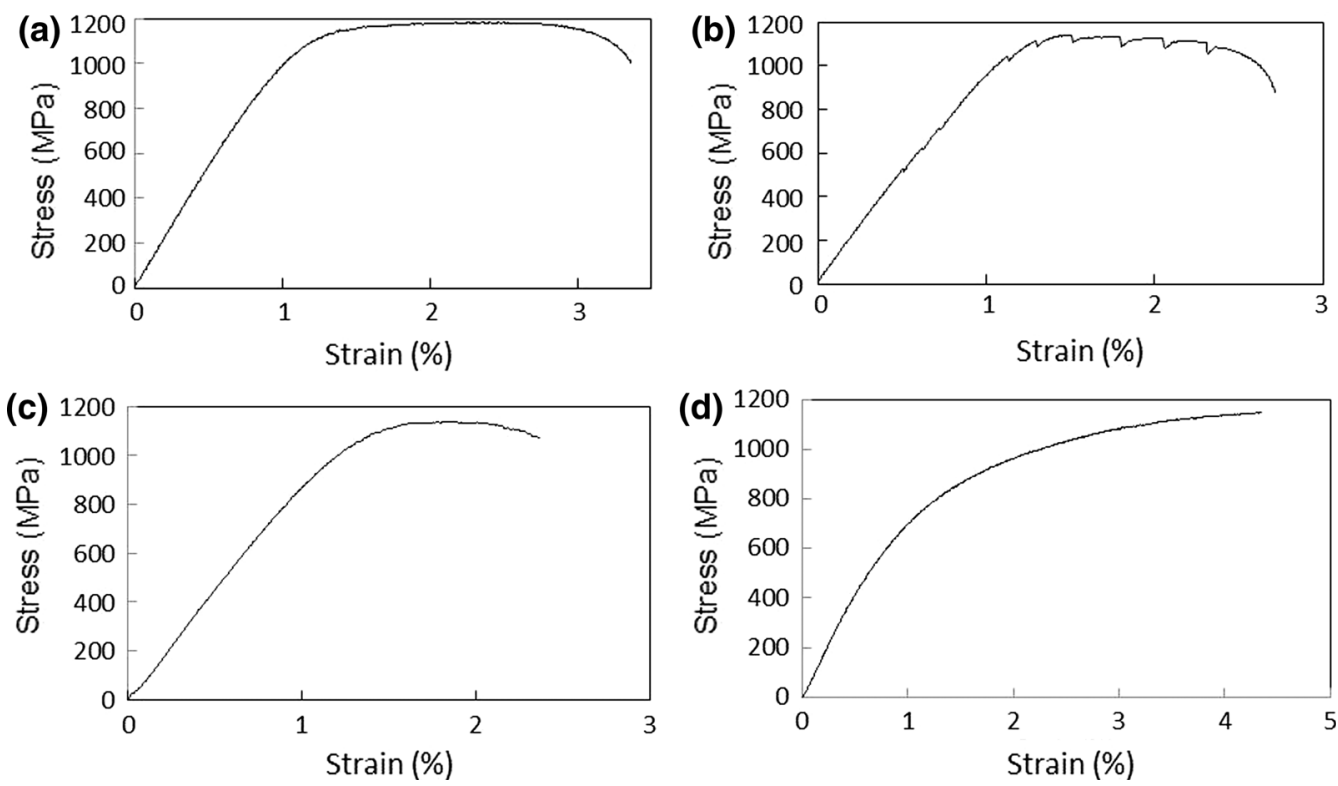

Fig. 3 Stress-strain curves of TRIP steel in martensite state: a without current; $\mathbf{b}$ single-pulse current; $\mathbf{c}$ multipulse current; $\mathbf{d}$ direct current

thermoelastic reversible martensitic transformation (TiNi), a current has to cause effect of hardening if $A_{\mathrm{s}}<T_{\text {def }}$ (Fig. 1) or softening if $T_{\text {def }}<A_{\mathrm{s}}[20,21]$. In the alloy with irreversible MT (TRIP steel), the current effect depends on the temperatures ratio $T_{\text {def }}$ and $M_{\mathrm{d}}$. A current has poor influences on deformation behavior at $T_{\text {def }}<M_{\mathrm{d}}$ (Fig. 2b) and also causes softening at $T_{\text {def }}>M_{\mathrm{d}}$ (Figs. 2c, d, 3).

\section{Conclusions}

1. View of tension stress-strain curve of TiNi alloy and TRIP steel strongly depends on the current modes and correlation of EPE and Joule heating. Shape memory and TRIP effects are suppressed for direct current mode, and stress-strain curves are typical ones as for stable austenite phase. The influence of single- or multipulse current is intermediate.

2. The tension of $\mathrm{Ti}_{49.3} \mathrm{Ni}_{507}$ alloy under direct current leads to the test temperature higher than $A_{\mathrm{f}}$ and an increase in stress level in the area of martensitic transformation.

3. The tension of austenite-martensite TRIP steel under without current or single current is the only one for a good combination of strength and plasticity. For all other current regimes, both the strength and plasticity decrease strongly.

Acknowledgments This work was supported by the Competitiveness Program of the National Research Nuclear University MEPhI (Moscow Engineering Physics Institute), contract with the Ministry of Education and Science of the Russian Federation No. 02.A03.21.0005, 27.08.2013, and RFBR (project \# 16-58-48001).

\section{References}

[1] O.A. Troitskiy, JETP Lett. 10, 18 (1969)

[2] K. Okazaki, M. Kagawa, H. Conrad, Scr. Metall. 12, 1063 (1978)

[3] H. Conrad, Mater. Sci. Eng., A 287, 276 (2000)

[4] O.A. Troitskiy, Yu.V. Baranov, Yu.S. Avraamov, A.D. Shlyapin, Phisical bases and technology of treatment of modern materials, V. 1, (Moscow-Izhevsk, Inst. Computer technologies, 2004) p.590

[5] A.F. Sprecher, S.L. Mannant, H. Conrad, Acta Metall. 34, 1145 (1986)

[6] V.V. Stolyarov, Bull. RAS Phys. 78, 234 (2014)

[7] R.S. Qin, Mater. Sci. Technol. 31, 203 (2015)

[8] K. Okazaki, M. Kagawa, H. Conrad, Mater. Sci. Eng. A 45, 109 (1980)

[9] W.A. Salandro, C. Bunget, L. Mears, Proc. ASME 573 (2011)

[10] R.S. Timsit, Scr. Metall. 15, 461 (1981)

[11] V. Stolyarov, Mater. Sci. Forum 584-586, 507 (2008)

[12] A.A. Potapova, N.N. Resnina, V.V. Stolyarov, J. Mater. Eng. Perform. 23, 2391 (2014)

[13] K.H. Lo, C.H. Shek, J.K.L. Lai, Mater. Sci. Eng., R 65, 39 (2009)

[14] M. Shirdel, H. Mirzadeh, M.H. Parsa, Mater. Character. 103, 150 (2015)

[15] H. Conrad, Mater. Res. Inn. 2, 1 (1998)

[16] M.L. Linderov, K. Zegel, AYu. Vinigradov, A. Vaidner, X. Birman, Vector of science. Tolyatti State Univer. 3, 208 (2013)

[17] X.L. Wu, M.X. Yang, F.P. Yuan, L. Chen, Y.T. Zhu, Acta Mater. 112, 337 (2016)

[18] M. Breda, F. Michieletto, E. Beridze, C. Gennari, Appl. Mech. Mater. 792, 568 (2015)

[19] J. Magargee, F. Morestin, J. Cao, J. Eng. Mater. Technol. 135, 041003 (2013)

[20] V. Stolyarov, U. Ugurchiev, Phys. High Pressure Technol. 19, 92 (2009)

[21] V. Stolyarov, Deformation behavior at rolling and tension under current in TiNi alloy. ESOMAT (2009). https://doi.org/10.1051/ esomat/200906033 